\title{
Treatment of Complex Extraperitoneal Appendiceal Abscesses Using a Single-Stage Laparoscopic Transabdominal Pre-Peritoneal Approach: A Description of Technique and Perioperative Care
}

\author{
Charalampos Seretis ${ }^{\mathrm{a}, \mathrm{b}}$, Fotios Seretis ${ }^{\mathrm{a}}$, Lucy Archer ${ }^{\mathrm{a}}$, Lida Lalou ${ }^{\mathrm{a}}$, Ali Mohammed Elhassan ${ }^{\mathrm{a}}$, \\ Leo Kretzmera, Paul Limª, Kassim Zayyan ${ }^{\mathrm{a}}$
}

\begin{abstract}
The surgical management of complicated appendicitis, especially when clinical diagnosis has been delayed substantially, can be challenging for the emergency general surgery team. The treatment plan needs to be tailored on an individual basis, with careful evaluation of the preoperative imaging studies, patients' prior intra-abdominal surgery and assessment for potential role of combining laparoscopic techniques with percutaneous interventions and/or open surgery. Herein, we present a clinical case with extensive extraperitoneal collections secondary to misdiagnosed appendicitis, which was treated using a single-stage laparoscopic transabdominal pre-peritoneal (TAPP) approach, bypassing the need for additional interventional radiology procedures or open surgeries, which are the most commonly used approaches in similar cases.
\end{abstract}

Keywords: Appendicitis; Psoas; Abscess; Laparoscopy; Endoscopy; TAPP

\section{Introduction}

Acute appendicitis is one of the most common general surgery emergencies, with the mainstay treatment being the performance of laparoscopic appendicectomy [1]. It is generally accepted that upon diagnostic doubt, the radiological examination of choice to confirm clinically suspected appendicitis is cross-sectional imaging covering the whole abdominopelvic

Manuscript submitted June 1, 2020, accepted June 12, 2020

Published online August 22, 2020

aDepartment of General Surgery, George Eliot Hospital NHS Trust, College Street, CV10 7DJ, Nuneaton, Warwickshire, West Midlands, UK

${ }^{b}$ Corresponding Author: Charalampos Seretis, Department of General Surgery, George Eliot Hospital NHS Trust, College Street, CV10 7DJ, Nuneaton, Warwickshire, West Midlands, UK. Email: babismed@gmail.com

doi: https://doi.org/10.14740/jcs414 compartment, usually in the form of computed tomography (CT) scan [2]. An additional benefit from preoperative crosssectional imaging includes the ability to assess the presence of intraperitoneal and/or extraperitoneal collections, which can be a sequel of long-standing inflammatory process in cases of delayed presentation to emergency surgery providing services. In patients with complicated collections as a result of acute appendicitis, especially when the collections extend in the extraperitoneal space, the general surgery team needs to consider the therapeutic approach with careful interpretation of crosssectional imaging bearing in mind the potential benefits and limitations of open, laparoscopic and percutaneous surgical interventions [3]. Herein, we present an interesting clinical case of misdiagnosed appendicitis with accompanying large extraperitoneal abscesses extending to the right retroperitoneum and space of Bogros, which was treated successfully laparoscopically using the transabdominal pre-peritoneal (TAPP) approach. We also describe potential alternative options within a context of minimally invasive interventions, which could be useful for the emergency general surgery teams which will encounter similar clinical scenarios.

\section{Case Report}

A 58-year-old Caucasian female was referred from her primary care physician to our emergency department for review by the on-call general surgery team, querying the presence of a strangulated right groin hernia. Her past medical and surgical history was unremarkable, however upon the initial consultation in the emergency department, she mentioned that approximately for over 1 month she had been experiencing pain in the right flank and right iliac fossa, and in addition she could intermittently feel a lump in the right inguinal region. She had been treated for urinary tract infection in the community during most of the above-mentioned period with partial only improvement of her symptoms. Upon clinical assessment, there was fullness in the right iliac fossa extending to the right inguinal region; however the overall features were not clinically consistent with presence of ventral and/or groin hernia, as suspected by the referring primary care physician. 

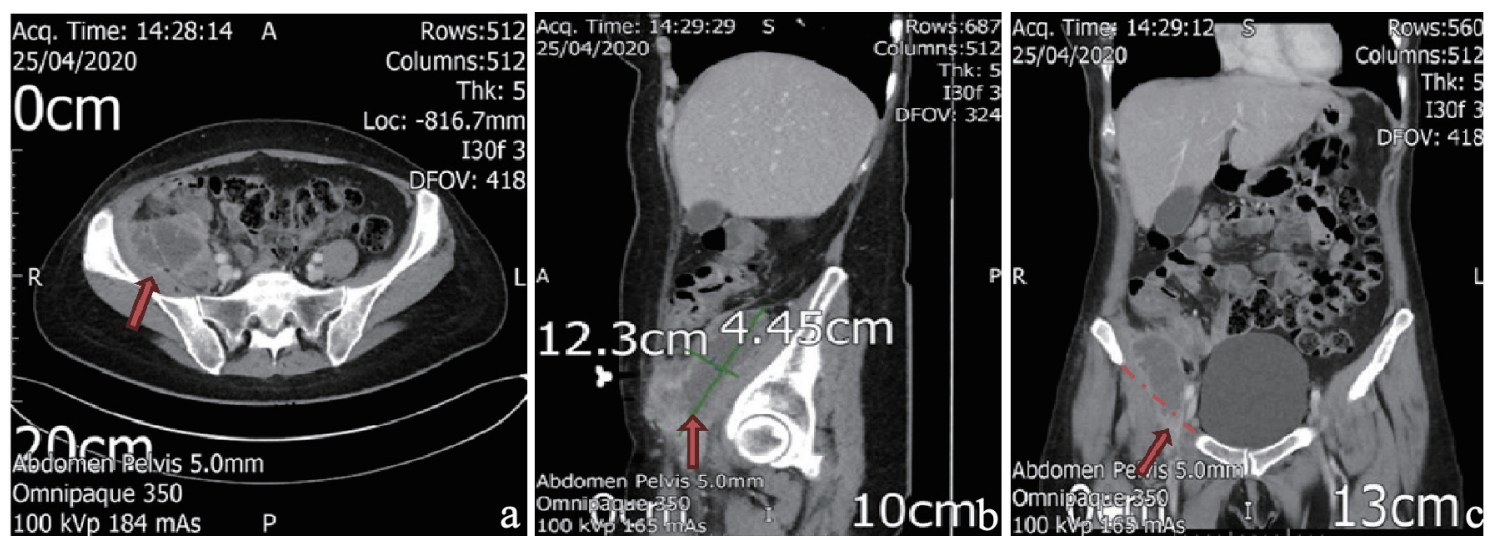

Figure 1. (a) Preoperative CT axial view of the collection at the level of the cecal pole. The trunk of the appendix cannot be identified. (b) Preoperative CT sagittal view of the extraperitoneal collection at its long axis $(12.3 \times 4.45 \mathrm{~cm})$. (c) Preoperative CT coronal view of the extraperitoneal collection (arrow), extending inferiorly to the level of inguinal ligament (interrupted line), which misled the primary care physician towards the diagnosis of a groin hernia rather than complicated intra-abdominal infection. CT: computed tomography.

Her baseline blood tests revealed substantially elevated inflammatory markers (C-reactive protein: $240 \mathrm{mg} / \mathrm{L}$ (reference value: $<11 \mathrm{mg} / \mathrm{L}$ ), white blood cell count: $20.86 \times 10^{9} / \mathrm{L}(\mathrm{ref}-$ erence value: $\left.4.00-11.00 \times 10^{9} / \mathrm{L}\right)$ ), and hence decision was to proceed with an urgent abdominopelvic CT scan, after the initial resuscitation with intravenous fluids and antibiotics. The performed CT scan revealed the presence of a large $12 \times 4.5$ $\mathrm{cm}$ multi-loculated collection arising from the cecal pole, extending to the right pre-peritoneal space below the level of the inguinal ligament and also tracking posteriorly and laterally to the psoas muscle; no other gross abnormality was detected and of note the appendix per se could not be identified (Fig. 1a-c). Reviewing the images and correlating with the patient's history, our impression was that the overall features corresponded to underlying complicated appendicitis, which had resulted in formation of the extensive extraperitoneal collections, as described above, due to substantial delays in establishing the diagnosis in primary care. After consultation with the patient, we opted to proceed with a diagnostic laparoscopy, appendicectomy and drainage of the extraperitoneal collections using a laparoscopic TAPP approach. We informed the patient of the increased likelihood of conversion to open surgery and potential need for ileocecal resection.

In the operating room, the patient was placed in supine position and the whole abdomen and pelvis were widely prepped and draped, with the right flank and right upper thigh being exposed as well, in case open access would be required. A vertical infraumbilical incision was made and access to the abdominal cavity was achieved using open cut-down technique under direct vision, allowing the insertion of a $12-\mathrm{mm}$ umbilical port. Two more 5-mm ports were inserted in the left iliac fossa and suprapubic regions under direct vision after the induction of pneumoperitoneum.

Upon the initial laparoscopic assessment, the cecal pole was plastered to the lateral abdominal wall; blunt dissection was used to gently reflect the cecum medially and the colic tenia coli fusion was used as a guide to locate the base of the appendix. A large volume of pus was aspirated when the appendiceal base was detached from the parietal peritoneum and the fistulating site leading towards the extraperitoneal collections was identified, measuring approximately $2 \times 2$ $\mathrm{cm}$. Regarding the appendix itself, it had completely disintegrated apart from its base $(2 \mathrm{~cm}$ in length), which enabled us to safely control it with placement of two Endoloop Vicryl sutures (number 0). Minor bleeding from the mesoappendix remnant site was controlled with placement of a Hem-O-Lok clip, after extension of the 5-mm left iliac fossa port to allow insertion of a blunt 11-mm port under direct vision. Upon the completion of the appendicectomy, we turned our attention to complete the TAPP drainage of the extraperitoneal collections. After insertion of another $5-\mathrm{mm}$ port in the right iliac fossa, we extended with electrocautery the opening of fistula orifice which connected the abdominal cavity with the extraperitoneal collection spaces, in a superior-lateral direction axis. Gentle external manual pressure was applied on the right groin and right flank, towards a cephalad direction, to facilitate further the evacuation of the extraperitoneal collections. Further volumes of pus were evacuated and thorough irrigation with warm saline was performed. Direct visualization of the extraperitoneal collection sites was achieved using our $30^{\circ}$ laparoscope and no obvious remaining purulent collections were identified. Once clear effluent was consistently obtained, two large-bore non-suction drains were inserted in the pelvis and in the right space of Bogros under laparoscopic guidance, and were exteriorized from the pelvic and right iliac fossa port sites respectively. Sheath closure of the $12-\mathrm{mm}$ midline and (extended) $11-\mathrm{mm}$ left iliac fossa port sites was performed with interrupted PDS (number 0) sutures using a J-shaped needle to prevent port-site hernia occurrence. The patient returned back to the ward for postoperative management and had an uneventful recovery. She was discharged on the fifth postoperative day with the drain, which was inserted in the extraperitoneal collection spaces in situ (the pelvic drain was removed $48 \mathrm{~h}$ post surgery), and was recalled on the 10th postoperative day for a repeat abdominopelvic CT scan to ensure complete resolution of all collec- 


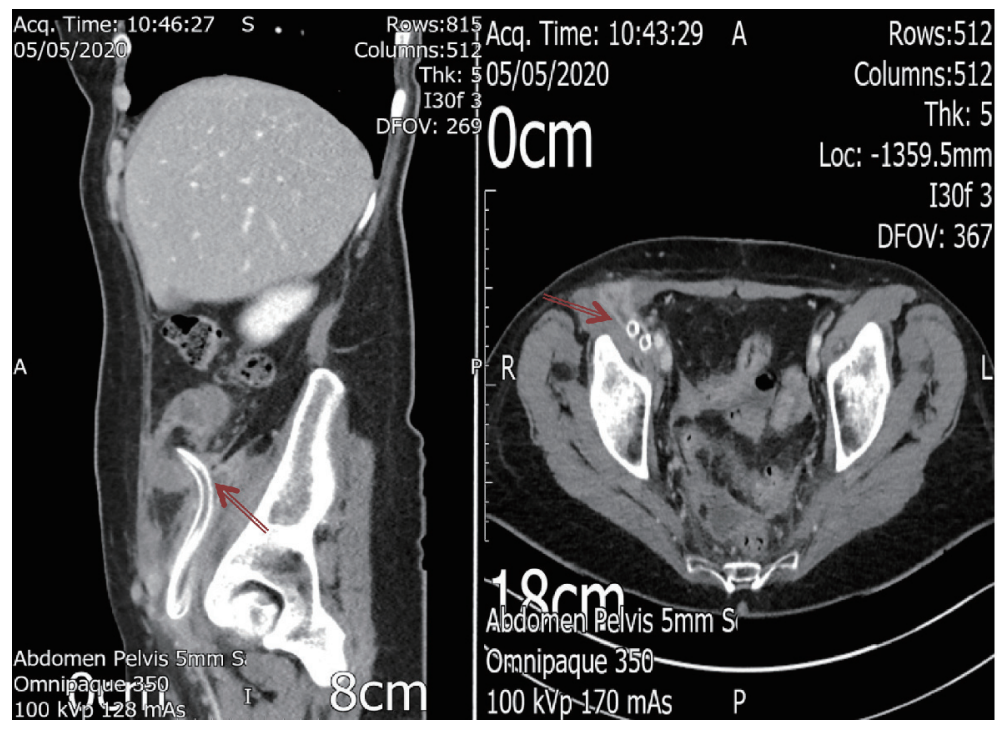

Figure 2. Postoperative CT coronal and axial views of the residual extraperitoneal collection (arrows), showing almost complete resolution; please note the kinking in the inferior aspect of the drain, which required re-adjustment under local anesthetic. Of note the residual collection was hemoserous fluid with no purulent features. CT: computed tomography.

tions. The repeat CT scan showed almost complete resolution of the extraperitoneal compartment abscesses, with overall good position of the drain, which was however kinked at its inferior aspect in the space of Bogros (Fig. 2). Under local anesthesia, the drain was readjusted appropriately and small volume of hemoserous fluid was further drained. The patient returned in our surgical assessment unit 5 days later and the drain was removed as total effluent was minimal. The histology was in consistency with acute appendicitis but as per institutional policy we arranged a follow-up colonoscopy at 2 months postoperatively to ensure absence of neoplastic lesion at the cecal pole, which could have triggered the onset of appendicitis.

\section{Discussion}

Extraperitoneal abscesses secondary to acute appendicitis are relatively uncommon and are usually a result of delayed presentation, missed diagnosis or in very rare occasions there could represent a fistulating process secondary to underlying appendiceal or cecal malignant tumors $[4,5]$. Although open surgery is the usually preferred approach to similar cases as patients often present with profound sepsis and quick control of the sepsis origin is required, there is an emerging role for minimally invasive surgery for the treatment of this group of patients [6]. Percutaneous approaches with the help of interventional radiology can be useful adjunct when drainage of these collections is needed, however this requires first of all the availability of the service per se, but also requires technical expertise, special equipment and depends on the suitability of the collections for percutaneous access (position, presence of multi-loculated collections). In addition, if a percutaneous approach is used, then the surgical team will not be able to obtain histology samples from the appendix or its remnant to rule out an appendiceal malignancy. Also, no assessment of the cecal pole, which sometimes is the site of malignant tumors resulting in appendicitis by obstruction of the appendiceal lumen, can be made without direct visualization.

For the above-mentioned reasons, and in order to avoid the prolonged recovery after major open surgery, we opted for the laparoendoscopic approach, consenting as well the patient for potential conversion to open surgery and possibility of postoperative percutaneous drainage if required. Although there are few cases in the literature describing similar laparoscopic approaches to proceed with these complicated appendicectomies with or without additional percutaneous drainage of the extraperitoneal collections by interventional radiology $[7,8]$, we are not aware of a previous description of a formal direct visualization and endoscopic drainage of the concurrent extraperitoneal collections using a formal TAPP approach. We believe that our technique is a safe and feasible option, as it is a one-stop procedure and bypasses the need for access to interventional radiology services. Also, it provides a quicker recovery pathway for the patient avoiding a major laparotomy, which is the usually preferred technique in the majority of the relevant presented cases. Finally, during the initial laparoscopy, we were able to locate the remnant of the appendiceal base, obtain safe control which prevents further complications related to future fecal fistulae and also sent the remaining part of the perforated appendix for histology. With respect to the postoperative care, we strongly advocate a limited pelvic CT scan prior to removal of the extraperitoneal drains to assess for the resolution of the drain collections and need to possibly readjust them, as we had to do in our case.

\section{Acknowledgments}

None to declare. 


\section{Financial Disclosure}

None to declare.

\section{Conflict of Interest}

None to declare.

\section{Informed Consent}

We hereby confirm that informed consent was obtained from the patient for publication of the case.

\section{Author Contributions}

$\mathrm{KZ}$ and CS contributed to the development of the presented technique concept. FS, LA, PL and AME contributed to the literature research and writing of manuscript. LL, LK and $\mathrm{CS}$ contributed to the editing and reviewing of the manuscript.

\section{Data Availability}

The authors declare that data supporting the findings of this study are available within the article.

\section{References}

1. Becker P, Fichtner-Feigl S, Schilling D. Clinical management of appendicitis. Visc Med. 2018;34(6):453-458.

2. Bax T, Macha M, Mayberry J. The utility of CT scan for the diagnostic evaluation of acute abdominal pain. Am J Surg. 2019;217(5):959-966.

3. de Jesus Lopes Filho G, Matone J, Arasaki CH, Kim SB, Mansur NS. Psoas abscess: diagnostic and therapeutic considerations in six patients. Int Surg. 2000;85(4):339343.

4. Al Shehri DM, Asiri AK, Abd El Maksoud WM. Late onset ilio-psoas abscess due to stump appendicitis: a case report. J Biomed Res. 2017;31(5):462-465.

5. Petrovic I, Pecin I, Prutki M, Augustin G, Nedic A, Gojevic A, Potocki K, et al. Thigh abscess as an extension of psoas abscess: the first manifestation of perforated appendiceal adenocarcinoma: case report. Wien Klin Wochenschr. 2015;127(15-16):645-648.

6. Moslemi S, Tahamtan M, Hosseini SV. A late-onset psoas abscess formation associated with previous appendectomy: a case report. Bull Emerg Trauma. 2014;2(1):55-58.

7. Otowa Y, Sumi Y, Kanaji S, Kanemitsu K, Yamashita K, Imanishi T, Nakamura T, et al. Appendicitis with psoas abscess successfully treated by laparoscopic surgery. World J Gastroenterol. 2014;20(25):8317-8319.

8. Choi SB, Han HJ, Kim WB, Song TJ, Choi SY. A case of a recurrent iliopsoas abscess masking a complicated appendicitis successfully treated by a laparoscopic approach. Surg Laparosc Endosc Percutan Tech. 2010;20(2):e69-72. 